\title{
Finite element developments for general fluid flows with structural interactions
}

\author{
Klaus-Jürgen Bathe ${ }^{1, *, \dagger}$ and Hou Zhang ${ }^{2}$ \\ ${ }^{1}$ Massachusetts Institute of Technology, 77 Massachusetts Avenue, Cambridge, MA, U.S.A. \\ ${ }^{2}$ ADINA R\&D, Inc. 71 Elton Avenue, Watertown, MA, U.S.A.
}

\begin{abstract}
SUMMARY
The objective in this paper is to present some developments for the analysis of Navier-Stokes incompressible and compressible fluid flows with structural interactions. The incompressible fluid is discretized with a new solution approach, a flow-condition-based interpolation finite element scheme. The high-speed compressible fluids are solved using standard finite volume methods. The fluids are fully coupled to general structures that can undergo highly non-linear response due to large deformations, inelasticity, contact and temperature. Particular focus is given on the scheme used to couple the fluid media with the structures. The fluids can also be modelled as low-speed compressible or slightly compressible media, which are important models in engineering practice. Some solutions obtained using ADINA are presented to indicate the analyses that can be performed. Copyright (C) 2004 John Wiley \& Sons, Ltd.
\end{abstract}

KEY WORDS: fluid flow; incompressible; compressible; FSI; ADINA

\section{INTRODUCTION}

The analysis of multiphysics problems, and specifically the solution of fluid-structure interactions, has been given increased attention during recent years [1]. This is largely because numerical methods have become very powerful and can be used at reasonable costs giving great benefits in scientific and engineering studies. Traditionally, fluid flows have been solved assuming rigid structures, and structures have been solved assuming fluid pressures. Sometimes iterations were used between the analyses of the two media to ensure that reasonable assumptions have been used in each case. However, there are many problems where a direct fully coupled analysis is needed to model the physics of the fluid-structure problem accurately. This is particularly the case when the structure undergoes large deformations in the interaction with the fluid and thermal effects need be included.

\footnotetext{
*Correspondence to: Klaus-Jürgen Bathe, Massachusetts Institute of Technology, 77 Massachusetts Avenue, Cambridge, MA 02139, U.S.A.

†E-mail: kjb@mit.edu
} 
Of course, fully coupled fluid-structure interactions in which the fluid is assumed to be an acoustic medium (with relatively small motions) have been analysed for some time and can now be solved very efficiently [2-4]. The assumption of small fluid particle motions can also be removed to allow in an effective way for actual fluid flows [5,6]. However, we are here concerned with the solution of the full Navier-Stokes (or Euler) equations for the fluid flow. There are numerous important applications where such flows are fully coupled with structures, meaning that the response in each medium is strongly affected by the response in the other, and it is important to predict the flows as well as the structural deformations accurately. We present some illustrative applications in Section 5.

Fluid flows are generally characterized as fully incompressible flows or high-speed compressible flows. In addition, it can be important to model flows as slightly compressible (reached from the fully incompressible case) and low-speed compressible (reached from the high-speed compressible case). These two flow models are frequently used in practice. Of course, structures are usually described by Lagrangian formulations, whereas fluids are described by Eulerian formulations. For the coupling of these media, it is common to use an arbitrary-LagrangianEulerian (ALE) formulation for the fluid [5,7]. Particular care must be taken to properly couple the fluid and the structure on the interface between the media. Also, an important ingredient of the overall solution scheme is the algorithm to update the fluid mesh which in the ALE formulation must move as the structure deforms.

In engineering practice and in scientific studies, it is clearly best to have a general approach towards solving fluid flows coupled with structures. The structural components might be shells with various possible behaviours [8] or solids that due to their low material stiffness are rather flexible. In each case, the structural deformations can be complex and highly affected by the specific fluid flow that takes place, and vice versa, the fluid flow can be highly affected by the structural deformations that occur.

Our objective in this paper is to present some developments to solve fluid flows with structural interactions. The flows can be modelled as mentioned above and of course can include effects of turbulence. The developments provide procedures for an analyst in engineering or the sciences to solve complex fluid flows with structural interactions.

In Section 2, we present a solution method for the laminar incompressible Navier-Stokes equations. An important point is that in engineering practice an analyst of a fluid flow structural interaction problem frequently does not know how high a Reynolds number flow will be encountered and what turbulence model should best be used. Hence, the problem is frequently best solved first by assuming laminar conditions. Then, continuing the analysis, the mathematical model might be (and frequently must be) refined by a suitable turbulence model. Of course, such hierarchical solution approach to a fluid-flow structural interaction problem (that is, using increasingly more complex mathematical models to describe the physics of the problem [9]) assumes that all models can actually be solved. For this reason, we have endeavored to develop a solution scheme that can solve the laminar Navier-Stokes equations at high Reynolds numbers, with reasonable accuracy, even when using relatively coarse meshes [10-12]. The flow-condition-based interpolation (FCBI) scheme that we are developing is a novel approach with good stability and accuracy characteristics [12]. The scheme can of course also be used for slightly compressible flows and low-speed compressible flows.

We then briefly mention in Section 3 the discretization scheme that we use for high-speed compressible flows $[13,14]$. 
These categories of modelling fluid flows are providing physically realistic mathematical models of many flow situations coupled with structural interactions.

The discretization schemes to solve these fluid models are used with an arbitrary-LagrangianEulerian formulation that is coupled to the Lagrangian formulation of structures $[15,16]$. These can undergo very large deformations, be subjected to non-linear material conditions, and contact. The finite element procedures used to model the structures in particular including contact conditions are described elsewhere $[9,17]$. Specifically, in fluid-structure interactions, frequently thin shell behaviour needs to be properly solved and therefore must be accurately represented $[18,19]$.

An important ingredient of the analyses is the scheme used to couple the structures and fluids. We describe this scheme in Section 4. This coupling is of course applicable to all the fluid flows mentioned above.

To demonstrate the use of the solution schemes, we present in Section 5 some illustrative example solutions obtained using ADINA, and finally, in Section 6, we give our concluding remarks.

\section{INCOMPRESSIBLE FLUID FLOWS}

We consider in this section the solution of the incompressible Navier-Stokes equations governing laminar flow. As we pointed out already, in practical fluid-structure interaction analyses, frequently, the Reynolds number of flow is not known prior to the analysis, and even if known, it is not clear what turbulence model to use. Hence, frequently, the analyst would like to obtain, in the first instance, a solution assuming laminar flow conditions. Once this solution has been calculated, assuming of course with reasonable accuracy, the fluid model can be refined by the use of an appropriate turbulence model.

In using such hierarchical models of fluid flows [9], it is important to recall that weak solutions exist of the laminar Navier-Stokes equations up to Reynolds numbers of about $10^{6}$ of course, provided transient analyses are considered and the initial conditions are sufficiently smooth [20,21]. Hence it is reasonable to seek finite element solutions of the laminar NavierStokes equations for high Reynolds number flows, even when using relatively coarse and unstructured meshes. Clearly, with such meshes it is hardly possible to have excellent accuracy in all aspects of the solution. Instead, the key aim must be to have in these solutions sufficient accuracy for the specific variables of interest [10,12]. The flow predictions reached with coarse (but still reasonable) meshes must be overall realistic and the forces transmitted to the structures must be of reasonable accuracy. The flow predictions should of course also converge with optimal rate to the 'exact' solution of the fluid flow mathematical model as the meshes are refined. Furthermore, the solution scheme should not be based on artificial solution parameters but be physically based, and numerically shown and mathematically proven to have the mentioned characteristics. These solution features are very difficult to reach in a solution scheme but are key to have a general and effective approach to solve fluid-flow structural interaction problems in engineering practice. The desired scheme does not exist as yet and can be considered to constitute an 'ideal' solution procedure [10-12]. We are developing the FCBI procedure to reach a method closer to this 'ideal' scheme.

Consider a general fluid flow problem governed by the incompressible Navier-Stokes equations. We assume that the problem is well posed in the Hilbert spaces $P$ and $V$. The governing equations in conservative form are 
Find the pressure $p(\mathbf{x}, t) \in P$, velocity $\mathbf{v}(\mathbf{x}, t) \in V$, and temperature $\theta(\mathbf{x}, t) \in V$ such that

$$
\begin{aligned}
& \frac{\partial \rho}{\partial t}+\nabla \cdot(\rho \mathbf{v})=0 \\
& \frac{\partial \rho \mathbf{v}}{\partial t}+\nabla \cdot(\rho \mathbf{v} \mathbf{v}-\tau)=\mathbf{f}^{\mathrm{B}} \\
& \frac{\partial \rho c_{v} \theta}{\partial t}+\nabla \cdot\left(\rho c_{v} \mathbf{v} \theta-k \nabla \theta\right)=2 \mu D^{2}+q^{\mathrm{B}}+\nabla \cdot \mathbf{v}(-p+\lambda \nabla \cdot \mathbf{v}) \\
& (\mathbf{x}, t) \in \Omega \times[0, T]
\end{aligned}
$$

where for fully incompressible flow, of course, the density $\rho$ is assumed to be constant, but for slightly compressible and low-speed compressible flows, we assume $\rho=\rho(p)$ and $\rho=\rho(p, \theta)$, respectively. Also, we assumed $c_{v}$ to be constant, an assumption that can be easily removed. The above equations are subject to the initial and boundary conditions

$$
\begin{aligned}
\mathbf{v}(\mathbf{x}, 0) & ={ }^{0} \mathbf{v}(\mathbf{x}) \\
\theta(\mathbf{x}, 0) & ={ }^{0} \theta(\mathbf{x}) \quad \mathbf{x} \in \Omega \\
p(\mathbf{x}, 0) & ={ }^{0} p(\mathbf{x}) \\
\mathbf{v} & =\mathbf{v}^{s} \quad(\mathbf{x}, t) \in \bar{S}_{v} \times(0, T] \\
\tau \cdot \mathbf{n} & =\mathbf{f}^{s} \quad(\mathbf{x}, t) \in S_{f} \times(0, T] \\
\theta & =\theta^{s} \quad(\mathbf{x}, t) \in \bar{S}_{\theta} \times(0, T] \\
k \mathbf{n} \cdot \nabla \theta & =q^{s} \quad(\mathbf{x}, t) \in S_{q} \times(0, T]
\end{aligned}
$$

where

$$
\begin{aligned}
\tau & =\tau(\mathbf{v}, p)=-p \mathbf{I}+2 \mu \mathbf{e}+\lambda \nabla \cdot \mathbf{v I} \\
D^{2} & =\mathbf{e} \otimes \mathbf{e} \equiv e_{i j} e_{i j}
\end{aligned}
$$

with the second viscosity $\lambda$ and

$$
\mathbf{e}=\frac{1}{2}\left[\nabla \mathbf{v}+(\nabla \mathbf{v})^{\mathrm{T}}\right]
$$

Also, $\mu$ is the viscosity, $k$ is the thermal conductivity, $\mathbf{f}^{\mathrm{B}}$ is the body force, $\Omega \in \mathbb{R}^{2,3}$ is a domain with the boundary $S=\bar{S}_{v} \cup S_{f}=\bar{S}_{\theta} \cup S_{q}\left(S_{v} \cap S_{f}=\emptyset, \quad S_{\theta} \cap S_{q}=\emptyset\right), T$ is the time span considered, $\mathbf{v}^{s}$ is the prescribed velocity on the boundary $\bar{S}_{v}, \mathbf{f}^{s}$ are the prescribed tractions on the boundary $S_{f}, \theta^{s}$ is the prescribed temperature on the boundary $\bar{S}_{\theta}, q^{s}$ is the prescribed heat flux on the boundary $S_{q}$, and $\mathbf{n}$ is the unit normal to the boundary.

The solution variables for these equations are the primitive variables; that is, the pressure $p$, velocity $\mathbf{v}$ and temperature $\theta$. For the finite element solution, we use a Petrov-Galerkin variational formulation with subspaces $V_{h}, U_{h}$ and $W_{h}$ of $V$, and $P_{h}$ and $Q_{h}$ of $P$ of the problem in Equations (1)-(6). The formulation for the numerical solution is: 


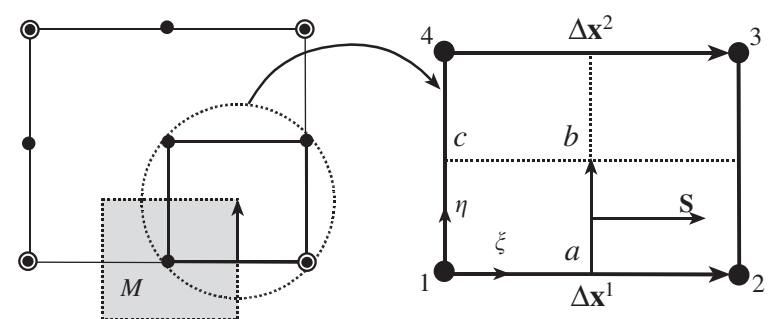

(a)

(b)

Figure 1. Control volume $M$ and evaluation of functions in $V_{h}$ for flux through $a b ; 0 \leqslant \xi, \eta \leqslant 1$ :

(a) two-dimensional 9-node element; and (b) sub-element.

Find $p \in P_{h}, \mathbf{v} \in V_{h}\left(R e_{h}\right), \phi \in V_{h}\left(P e_{h}\right)$ and $\mathbf{u}, \theta \in U_{h}$ such that for all $w \in W_{h}, q \in Q_{h}$

$$
\begin{array}{r}
\int_{\Omega} q\left[\frac{\partial \rho}{\partial t}+\nabla \cdot(\rho \mathbf{u})\right] \mathrm{d} \Omega=0 \\
\int_{\Omega} w\left[\frac{\partial \rho \mathbf{u}}{\partial t}+\nabla \cdot(\rho \mathbf{u v}-\tau(\mathbf{u}, p))-\mathbf{f}^{\mathrm{B}}\right] \mathrm{d} \Omega=\mathbf{0} \\
\int_{\Omega} w\left[\frac{\partial \rho c_{v} \theta}{\partial t}+\nabla \cdot\left(\rho c_{v} \mathbf{u} \phi-k \nabla \theta\right)-2 \mu D^{2}-q^{\mathrm{B}}-\nabla \cdot \mathbf{u}(-p+\lambda \nabla \cdot \mathbf{u})\right] \mathrm{d} \Omega
\end{array}
$$

where $R e_{h}$ and $P e_{h}$ are the element Reynolds and element Peclet numbers, respectively. We should note the use of the different spaces in the above discretization scheme. The trial functions in $U_{h}$ are the usual functions of finite element interpolations for velocities and temperature [9]. Similarly, the interpolation functions in the space $P_{h}$ are the usual functions used in incompressible analysis to satisfy, for the selected velocity interpolations, the inf-sup condition of incompressible analysis $[9,22]$. Hence, various suitable function spaces $U_{h}$ and $P_{h}$ are available.

An important ingredient of the scheme is that the trial functions in $V_{h}$ are different from the functions in $U_{h}$. They are defined using the flow conditions along the sides/faces of control volumes around the element nodes. Specifically, the trial functions in $V_{h}\left(R e_{h}\right)$ and $V_{h}\left(P e_{h}\right)$ are determined, respectively, based on the element Reynolds numbers (for velocity) and element Peclet numbers (for temperature). For example, considering a 9-node element and the control volume shown in Figure 1 and linear functions in $U_{h}$, we would use for $V_{h}\left(R e_{h}\right)$ the functions

$$
\left[\begin{array}{ll}
h_{1}^{v} & h_{4}^{v} \\
h_{2}^{v} & h_{3}^{v}
\end{array}\right]=\left[\mathbf{h}\left(x^{1}\right), \mathbf{h}\left(x^{2}\right)\right] \mathbf{h}(\eta) \mathbf{h}^{\mathrm{T}}(\eta)
$$

with

$$
x^{k}=\frac{\mathrm{e}^{q^{k} \xi}-1}{\mathrm{e}^{q^{k}}-1}, \quad q^{k}=\frac{\rho \overline{\mathbf{u}}^{k} \cdot \Delta \mathbf{x}^{k}}{\mu}
$$


where $\overline{\mathbf{u}}^{k}$ is the flow solution evaluated at the centre of the sides considered $\left(\xi=\frac{1}{2}\right)$ and $\eta=0,1$ for $k=1,2$, respectively).

Finally, the elements in the spaces $Q_{h}$ and $W_{h}$ are step functions. For example, for the node considered in Figure 1, we have for the element in $W_{h}$,

$$
h_{1}^{w}= \begin{cases}1 & (\xi, \eta) \in\left[0, \frac{1}{2}\right] \times\left[0, \frac{1}{2}\right] \\ 0 & \text { else }\end{cases}
$$

Of course, the trial functions in $U_{h}$ and $V_{h}$ are 'attached' to the same nodal velocities and temperatures. A formulation example is presented in Reference [12] but of course other elements with appropriate interpolations could be used.

The stability in the solution is obtained through three ingredients in the scheme:

- The use of the pair of functions in $U_{h}$ and $P_{h}$ to satisfy the inf-sup condition of incompressible analysis. These are the same pairs of functions that are, for example, also used in the analysis of solids or acoustic fluids [9, 22, 23].

- The use of step weight functions around nodes, which results in a strong local mass and momentum conservation, and hence directly also in the global mass and momentum conservation.

- The use of the flow-condition-based interpolation in the space $V_{h}$, which results in stability of the convective terms (the 'upwinding effect' which is introduced in a natural and physically based manner).

Considering the effectiveness of the scheme, it is important to note that the use of the trial functions makes it possible to calculate consistent Jacobian matrices in the Newton-Raphson iterations to solve the algebraic finite element equations [9]. Hence consistent stiffness matrices can be established for the complete fluid-structure system which makes it possible to solve complex practical problems with highly non-linear response.

We discussed above the solution of the governing equations assuming laminar flow conditions. To introduce turbulence models, these equations are supplemented by the governing equations of the turbulence models. The solution of the convective-diffusive equations governing the turbulence effects is then obtained using the same numerical scheme.

While we use the current FCBI scheme already effectively in solutions, see Reference [12] and Section 5, clearly further improvements based on physical insight and mathematical analysis are very desirable-and we believe can also be reached, because the solution approach can be explored much further.

\section{HIGH-SPEED COMPRESSIBLE FLUID FLOWS}

The governing Navier-Stokes equations in high-speed compressible flow are written in their strong conservation form

$$
\frac{\partial \mathbf{U}}{\partial t}+\nabla \cdot \mathbf{F}=\mathbf{S}
$$


with

$$
\mathbf{U}=\left[\begin{array}{c}
\rho \\
\rho \mathbf{v} \\
\rho E
\end{array}\right], \quad \mathbf{F}=\left[\begin{array}{c}
\rho \mathbf{v} \\
\rho \mathbf{v} \mathbf{v}-\tau \\
\rho \mathbf{v} E-\tau \cdot \mathbf{v}-k \nabla \theta
\end{array}\right], \quad \mathbf{S}=\left[\begin{array}{c}
0 \\
\mathbf{f}^{\mathrm{B}} \\
\mathbf{f}^{\mathrm{B}} \cdot \mathbf{v}+q^{\mathrm{B}}
\end{array}\right]
$$

where $E\left(=\frac{1}{2} \mathbf{v} \cdot \mathbf{v}+e\right)$ is the specific energy and $e$ is the internal energy.

The solution variables are now the conservative variables; that is, the density $\rho$, the specific discharge $\rho \mathbf{v}$ and the specific total energy $\rho E$. Various boundary conditions are now possible which depend on whether external or internal flows are considered, whether subsonic or supersonic inlet/outlet conditions are present, and so on, see Reference [14] for a table giving boundary conditions.

To ensure (strongly) local conservation of mass, momentum and energy, we still use the step functions as weighting functions. However, we also use a flux-splitting method to evaluate the total fluxes along control volume sides/faces of the element nodes in order to capture shocks in flow solutions. Details of the solution scheme can be found in Reference [13].

\section{STRUCTURES AND THE COUPLING BETWEEN FLUID FLOWS AND STRUCTURAL MEDIA}

As we mentioned above, the structural domains are described using a Lagrangian formulation [9]. The structures can comprise solids, shells, beams, and other structural elements, including contact surfaces. Of particular interest in fluid-structure interactions is the capability to analyse thin shells interacting with fluid flows. Thin shell structures display a variety of deformation behaviours $[8,24]$ and the analysis of their interactions with fluid flows can be a significant challenge. In particular, an unstable chaotic response of the structure might occur that is important to predict $[16,25,26]$.

The coupling of the fluid and structural response can be achieved numerically in different ways, but in all cases, of course, the conditions of displacement compatibility and traction equilibrium along the structure-fluid interfaces must be satisfied, see e.g. References [13-16].

Displacement compatibility:

$$
\mathbf{d}_{\mathrm{f}}=\mathbf{d}_{\mathrm{s}} \quad(\mathbf{x}, t) \in S_{\mathrm{i}} \times[0, T]
$$

Traction equilibrium:

$$
\mathbf{f}_{\mathrm{f}}=\mathbf{f}_{\mathrm{s}} \quad(\mathbf{x}, t) \in S_{\mathrm{i}} \times[0, T]
$$

where $\mathbf{d}_{\mathrm{f}}$ and $\mathbf{d}_{\mathrm{s}}$ are the displacements, $\mathbf{f}_{\mathrm{f}}$ and $\mathbf{f}_{\mathrm{s}}$ are the tractions of the fluid and solid, respectively, and $S_{\mathrm{i}}$ is the interface of the fluid and solid domains.

These conditions must be imposed efficiently in the numerical solution. Assume that the fluid and structural domains have been meshed using entirely different elements (see Figure 2). We consider any well-posed structural and fluid models associated with any physically realistic material and boundary conditions. 


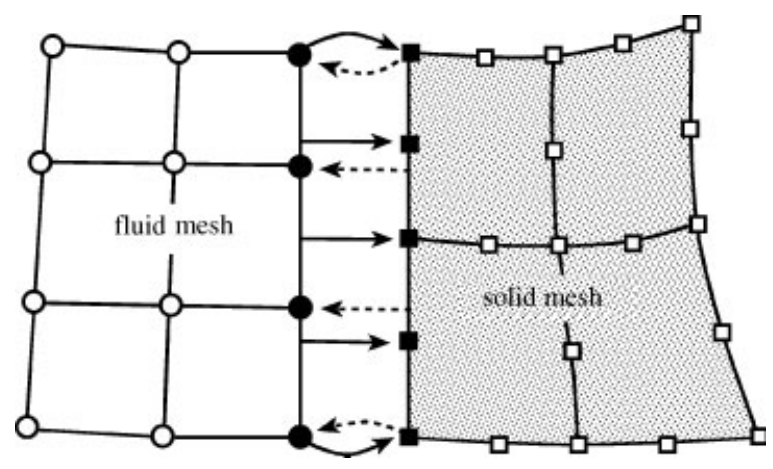

Figure 2. Illustration of fluid and solid meshes and mappings of displacements and tractions on fluid-structure interfaces. The solid arrows indicate traction mapping and the dashed arrows indicate displacement mapping.

The conditions of stress equilibrium require that, on an interface, the stresses of the fluid (represented by fluid variables defined at the fluid mesh points) be balanced by the stresses in the structure. Thus, we map the fluid tractions onto the structural nodes and then calculate the corresponding forces along the structural interface

$$
\mathbf{F}^{S}(\mathbf{v}, p)=\int_{S_{\mathrm{i}}} \mathbf{H}^{S^{\mathrm{T}}} \hat{\mathbf{f}}_{\mathrm{f}} \mathrm{d} S
$$

where $\hat{\mathbf{f}}_{\mathrm{f}}$ denotes the fluid tractions at the location of the structural nodes and the matrix $\mathbf{H}^{S}$ stores the displacement interpolation functions of the structural elements along the interface. The nodal forces calculated in Equation (20) are applied to the structural model as external forces. This procedure ensures 'consistency' in the stress transfer between the fluid and structure (that is, the patch test is passed [9]).

In order to determine the fluid mesh nodal displacements, we first consider the boundary nodes on the fluid-structure interfaces. Here the Lagrangian co-ordinates are used to impose the displacement compatibility condition (18). We enforce the solid displacements onto the fluid nodes by using the displacement interpolations on the solid. Of course, on the rest of the boundary, the fluid nodal co-ordinates can be moved or be fixed (independent of the fluid velocity) but the geometric domain of the fluid must not change. To ensure regularity of the mesh, Laplace equations are solved, first over internal and boundary lines, then over surfaces, and finally over volumes.

Using this approach, the displacements along the interfaces are (in discretized form) compatible between the fluid and solid domains. In the continuum, the displacements, velocities and accelerations are compatible. To achieve this compatibility directly in the numerical solution, our approach is to simply use one integration scheme (in ADINA, the first and second order implicit Runge-Kutta methods) for the complete coupled system.

Using the above discretization schemes, the resulting coupled algebraic system of equations to be solved for each time step considered can be written as

$$
\mathbf{F}(\mathbf{X})=\left[\begin{array}{l}
\mathbf{F}_{\mathrm{f}}\left(\mathbf{X}_{\mathrm{f}}, \mathbf{X}_{\mathrm{s}}\right) \\
\mathbf{F}_{\mathrm{s}}\left(\mathbf{X}_{\mathrm{f}}, \mathbf{X}_{\mathrm{s}}\right)
\end{array}\right]=\mathbf{0}
$$


where, respectively, $\mathbf{F}_{\mathrm{f}}$ and $\mathbf{F}_{\mathrm{s}}$ represent the equation systems for the fluid and solid models, and $\mathbf{X}_{\mathrm{f}}$ and $\mathbf{X}_{\mathrm{s}}$ are the fluid and solid nodal solution variables. In short, $\mathbf{F}$ represents the coupled system of equations and $\mathbf{X}$ represents all nodal solution variables of the coupled system. The non-linear system of equations can be solved either directly as one set or iteratively by partitioning [16,27, 28].

In the 'directly fully coupled solution' using, for example, the Newton-Raphson method, the linearization of the equations at each iteration gives

$$
\left[\begin{array}{ll}
\mathbf{A}_{\mathrm{ff}} & \mathbf{A}_{\mathrm{fs}} \\
\mathbf{A}_{\mathrm{sf}} & \mathbf{A}_{\mathrm{ss}}
\end{array}\right]\left[\begin{array}{c}
\Delta \mathbf{X}_{\mathrm{f}} \\
\Delta \mathbf{X}_{\mathrm{s}}
\end{array}\right]=\left[\begin{array}{c}
-\mathbf{F}_{\mathrm{f}}\left(\mathbf{X}_{\mathrm{f}}, \mathbf{X}_{\mathrm{s}}\right) \\
-\mathbf{F}_{\mathrm{s}}\left(\mathbf{X}_{\mathrm{f}}, \mathbf{X}_{\mathrm{s}}\right)
\end{array}\right]
$$

These equations are solved directly with a sparse solver or iteratively [9] and the new solution vector is updated by

$$
\mathbf{X} \Leftarrow \mathbf{X}+\Delta \mathbf{X}
$$

The Jacobian matrices are calculated using [9]

$$
\begin{array}{ll}
\mathbf{A}_{\mathrm{ff}}=\frac{\partial \mathbf{F}_{\mathrm{f}}}{\partial \mathbf{X}_{\mathrm{f}}}, & \mathbf{A}_{\mathrm{fs}}=\frac{\partial \mathbf{F}_{\mathrm{f}}}{\partial \mathbf{X}_{\mathrm{s}}} \\
\mathbf{A}_{\mathrm{sf}}=\frac{\partial \mathbf{F}_{\mathrm{s}}}{\partial \mathbf{X}_{\mathrm{f}}}, & \mathbf{A}_{\mathrm{ss}}=\frac{\partial \mathbf{F}_{\mathrm{s}}}{\partial \mathbf{X}_{\mathrm{s}}}
\end{array}
$$

In the 'iterative solution by partitioning', the fluid equations and solid equations are solved alternately (using direct or iterative methods as well), to pass latest solutions from one model to the other. In each case the iteration is continued until the coupled system (21) has been solved up to a given tolerance.

Note that in this approach, the fluid stresses are imposed as forces onto the structure, and the structural displacements impose a change onto the fluid domain. We could have proceeded in the opposite way, that is, by imposing stress boundary conditions onto the fluid and velocity boundary conditions onto the structure. However, this approach might be unstable for general fluid-structure interaction analysis, because any small error in displacements imposed onto the structure by the fluid velocities will result into large errors in tractions imposed onto the fluid.

Other approaches are to consider the complete structural and fluid system using the methods described in References [29,30]. However, it is not yet clear how these methods will perform in engineering practice when the stresses in the structure need be calculated accurately with very large structural displacements.

An important requirement in the analysis of a non-linear dynamic system can be to assess whether a stable or unstable, notably chaotic, response has been predicted. In the case of a fluid-structure system we would usually be interested in characterizing the structural response, because it is the structure that should not become unstable.

A rather obvious way to assess the character of the calculated response is to perform a Fourier analysis of the solution calculated at a particular point of the structure. However, the 
specific point needs to be selected, the assessment would only be carried out in post-processing of the response, and the solution at the point may not properly represent the global response of the structure (and indeed the convergence at a point may not even be defined [24]). Hence, a global measure of the character of the solution should be calculated and we have developed a scheme that uses the Lyapunov exponent for that purpose $[16,25,26]$. In this scheme, during the non-linear response solution, it is assessed whether a perturbation in the response will grow as given by the Lyapunov exponent. The added computational expense is relatively small and a global measure of the stability of the structural system acted upon by the fluid is directly calculated. Of course, the scheme does have limitations in that, for example, only one exponent is calculated and only the long-term instability is measured, as pointed out in References [16, 25, 26], see also Section 5.4.

\section{ILLUSTRATIVE SOLUTIONS}

The objective in this section is to present some solutions that briefly demonstrate the analysis capabilities described above. In each analysis case, a fluid flow structural interaction solution is considered.

(a)
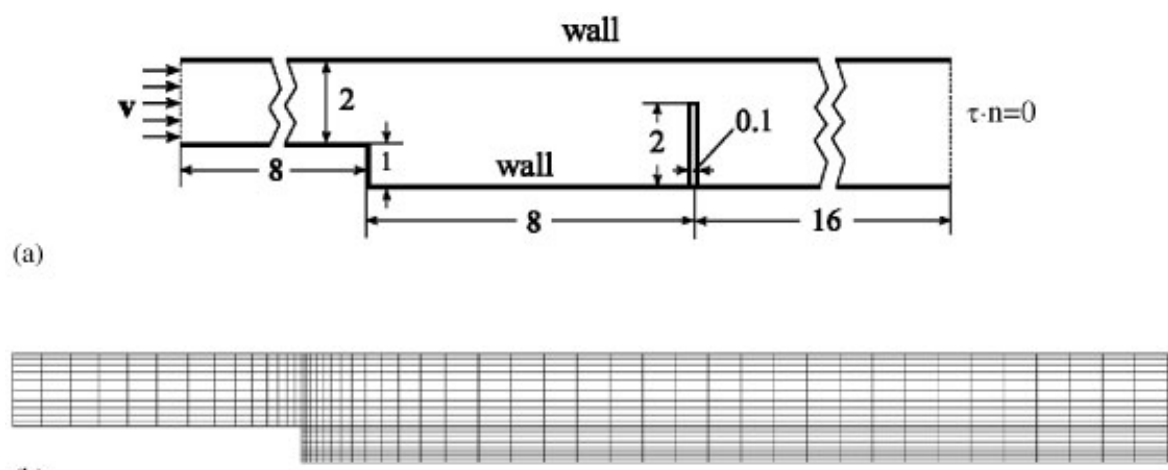

(b)

Figure 3. (a) Sketch of the flow over backward facing step problem, cantilever in channel is not present in the first analysis; (b) fluid mesh of four-node elements [12,19] used in the flow over backward facing step; (c) velocity fields and streamlines using laminar model in the flow over backward facing step: (1) $R e=1$; (2) $R e=10^{2}$; (3) $R e=10^{4}$; (4) $R e=10^{6}$; (d) velocity fields and streamlines using turbulence model in the flow over backward facing step: (1) $R e=1$; (2) $\operatorname{Re}=10^{2}$; (3) $\operatorname{Re}=10^{4}$; (4) $R e=10^{6}$; (e) computed solutions compared with the experimental data [31]: (1) $x$-velocity profile at location $x=4$; (2) $x$-velocity profile at location $x=6$; (f) fluid mesh used in the flow over backward facing step and a cantilever. The cantilever is modelled using 9-node 2D solid elements with $E=10^{7}$ and $v=0.3$; (g) velocity fields obtained using laminar model in the flow over backward facing step and a cantilever. Case (1): $\operatorname{Re}=1(v=1, \mu=1, \rho=0.5)$; Case (2): $\operatorname{Re}=100(v=1$, $\mu=1, \rho=50$ ). Horizontal displacement $d_{x}$ at tip of cantilever, Case (1): $d_{x}=0.093$; Case (2): $d_{x}=0.472$; (h) velocity fields obtained using turbulence model in the flow over backward facing step and a cantilever. Case (1): $\operatorname{Re}=1000(v=1, \mu=0.1, \rho=50), d_{x}=0.366$; Case (2): $\operatorname{Re}=10000$

$$
(v=1, \mu=0.01, \rho=50), d_{x}=0.365 \text {. }
$$


(1)

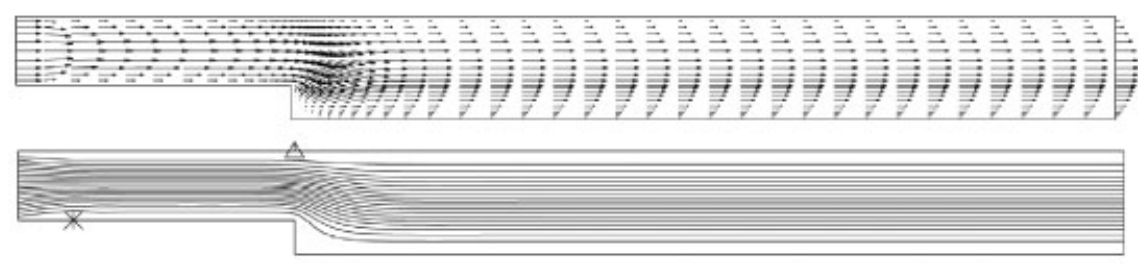

(2)

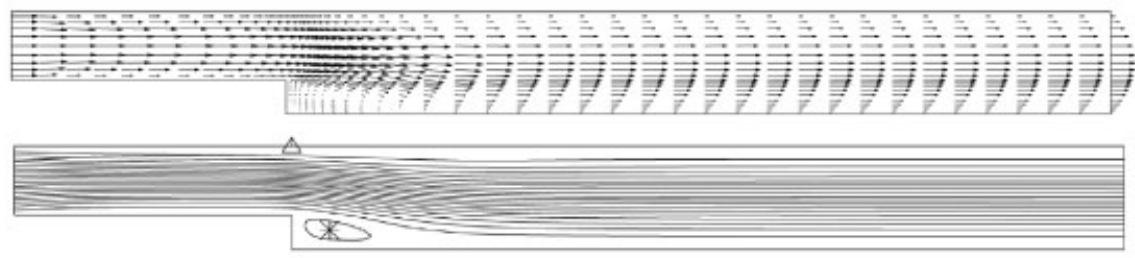

(3)

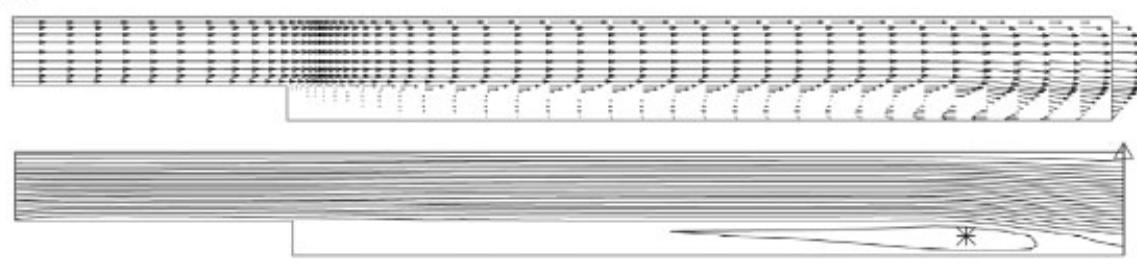

(4)

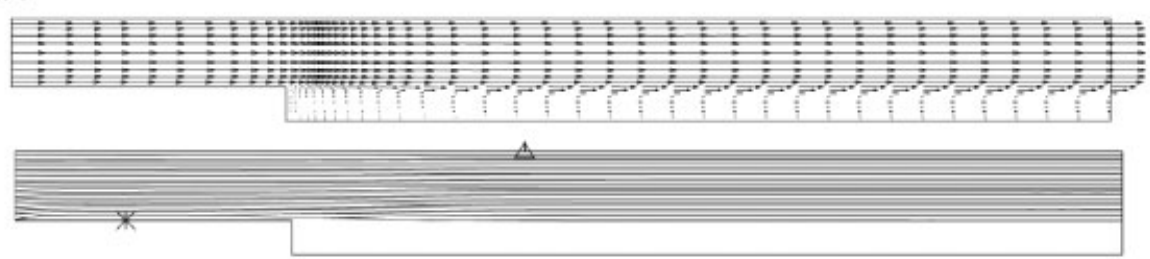

(c)

Figure 3. Continued.

\subsection{Flow over backward facing step problem}

We consider the fluid flow problem shown in Figure 3(a). This is a well-known problem, involving turbulent flow, used to test numerical solution schemes because experimental results are available. We first consider the flow problem without structural interaction, and then modify the problem to include a structural response. 
(1)

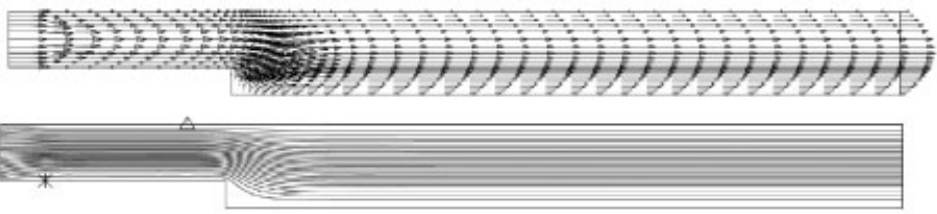

(2)

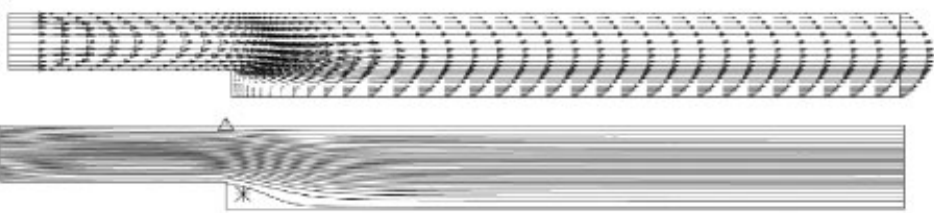

(3)

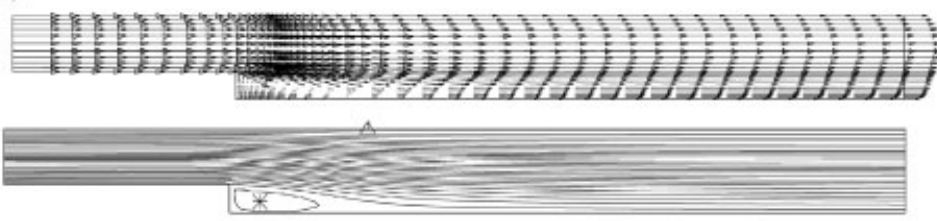

(4)

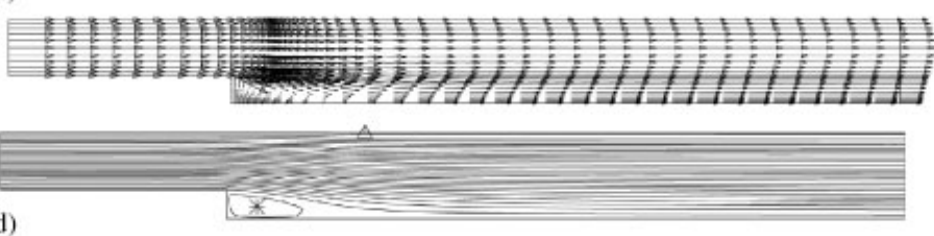

(1)

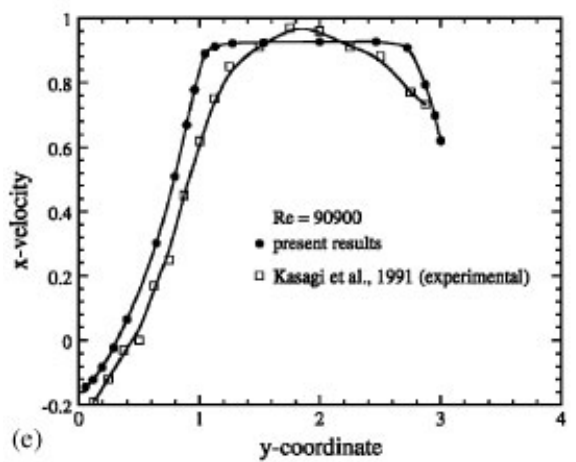

(2)

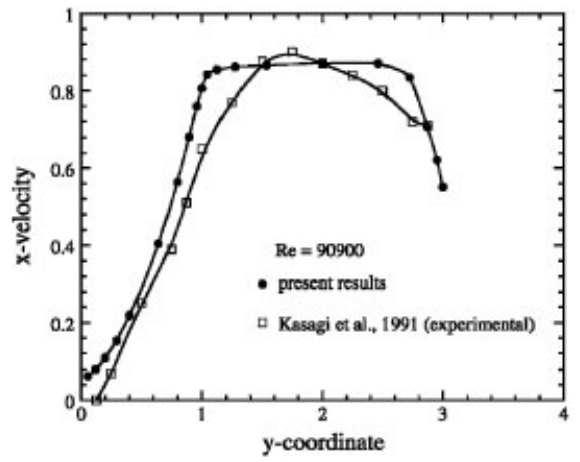

Figure 3. Continued.

Our first purpose is to illustrate the approach described in the paper towards the solution of incompressible fluid flow problems; namely that an analyst may well desire first a laminar flow solution and then refine the flow model to include an appropriate turbulence model. 


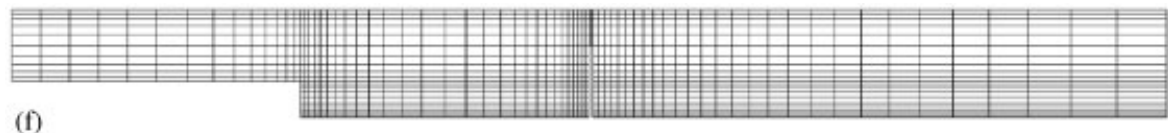

(f)

(1)

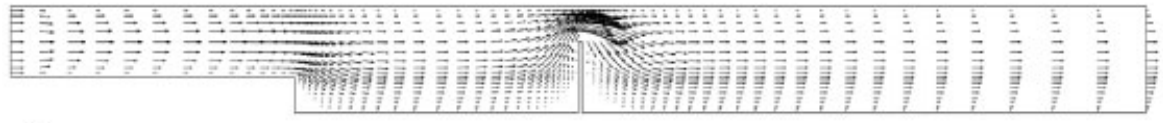

(2)

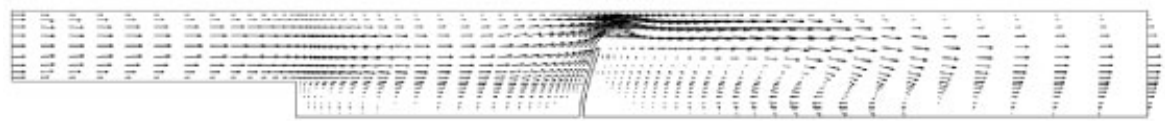

(g)

(1)

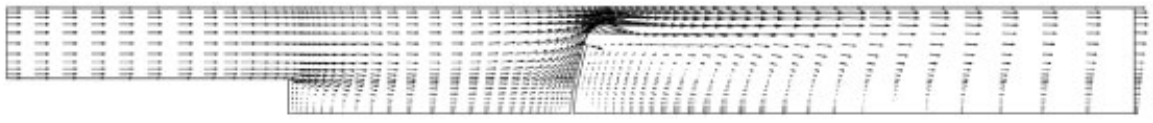

(2)

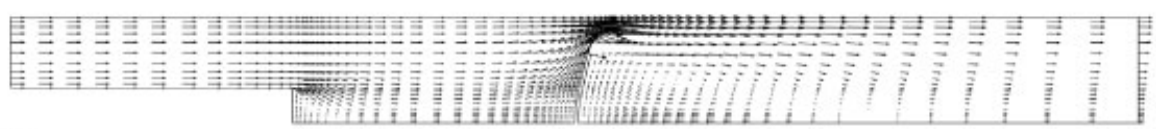

(h)

Figure 3. Continued.

Figure 3(b) shows the mesh used and Figures 3(c) and 3(d) give the laminar and turbulent flow solutions respectively for $R e=1,10^{2}, 10^{4}$ and $10^{6}$ (by fixing the inlet velocity and changing the viscosity). We used the standard $K / \varepsilon$ model to include the turbulence effects. Of course, at low Reynolds numbers the same flow is predicted with or without the turbulence model. Then, as the Reynolds number is increased, it becomes important to include turbulence effects in the solution in order to obtain meaningful results. Figure 3(e) shows some computed results compared with experimental results at $R e=90900$ (of course, including the turbulence effects).

In Figure 3(c), we give the laminar flow results for very high Reynolds numbers. This is-of course-merely a numerical test of the solution scheme and indeed it is a rather 'drastic' test of the FCBI solution scheme used.

We next place a flexible cantilever beam into the channel. Figure 3(f) shows the fluid mesh and Figures 3(g) and 3(h) give the computed results for different Reynolds numbers using 

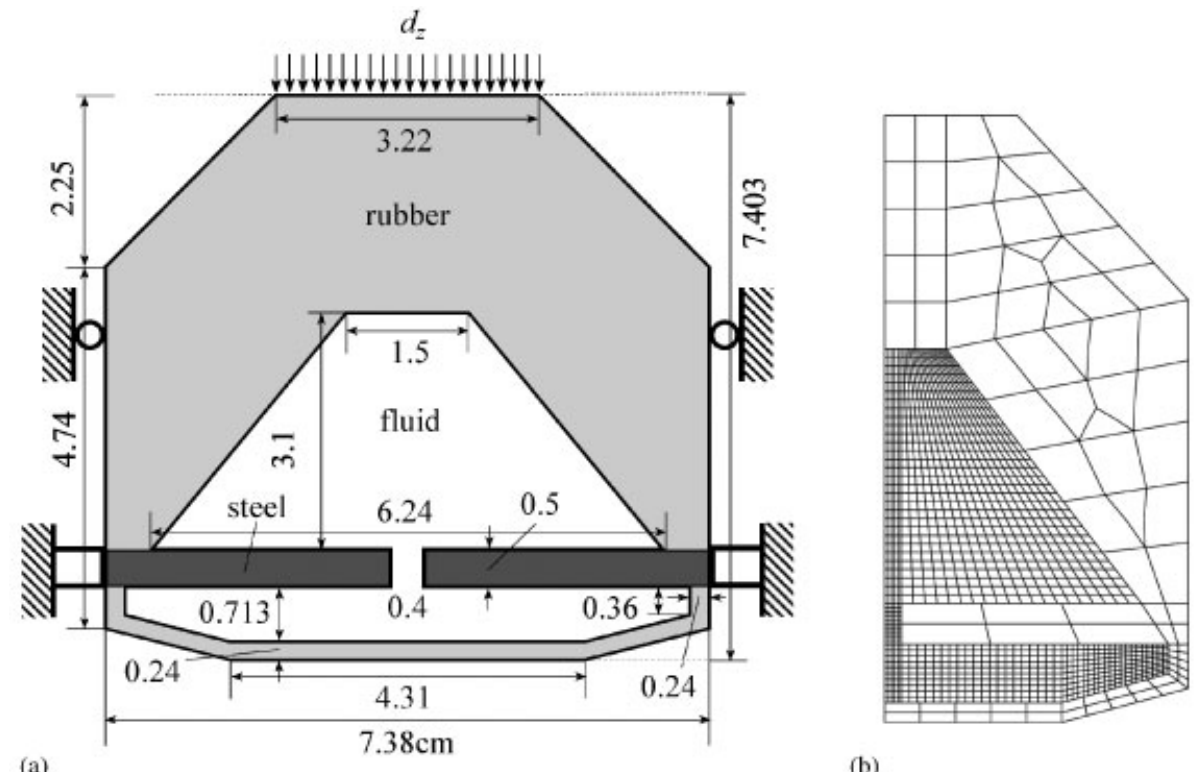

(b)

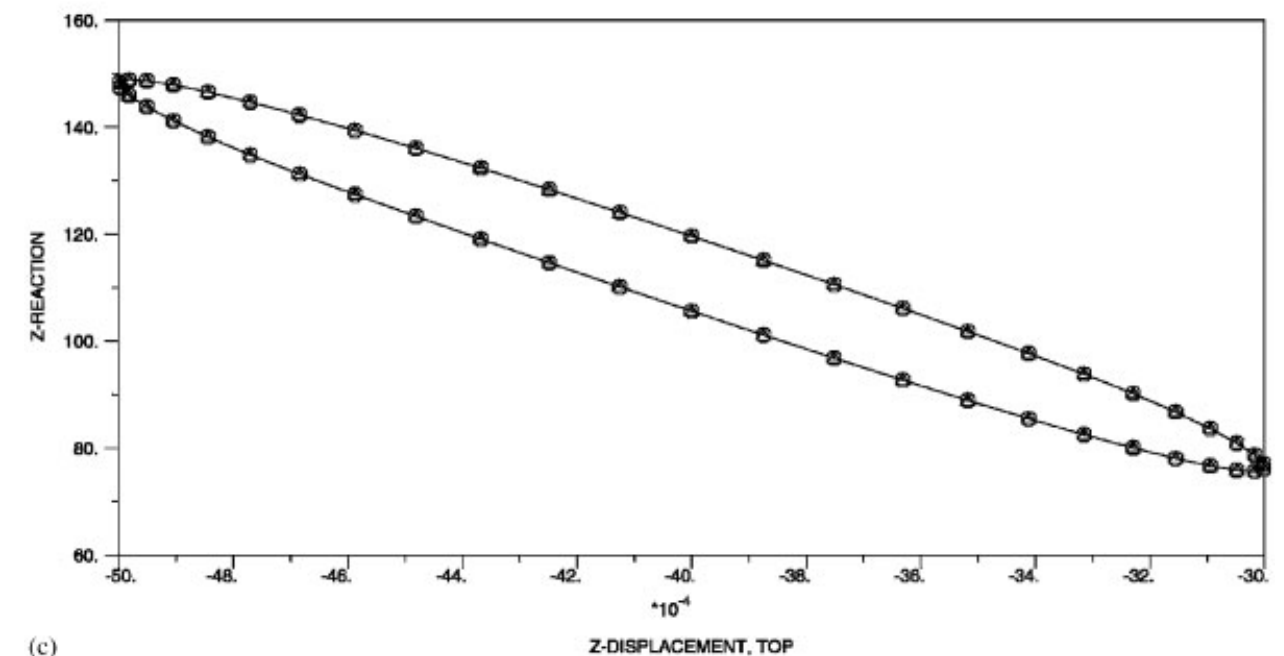

Figure 4. (a) Definition of the hydraulic engine mount problem. The displacement at the top is prescribed as $d_{z}=-0.4+0.1 \sin (20 \pi t)(\mathrm{cm})$; (b) fluid mesh (four-node elements) and solid mesh (9/3 elements [9]) used in the hydraulic engine mount problem; and (c) Z-reaction force (N) at the mounted location verses the $z$-displacement $(\mathrm{m})$ prescribed at the top.

laminar and turbulent flow assumptions (both the fluid density and the viscosity are changed, for the purpose of calculating structural deformations under different fluid pressures). For the higher $R e$ values the $K / \varepsilon$ model is used. Of course, the flow is now showing a totally different behaviour due to the obstruction by the cantilever. 
Table I. Material data used for hydraulic engine mount problem.

\begin{tabular}{lccc}
\hline Oil & Steel & \multicolumn{2}{c}{ Rubber (Ogden model) } \\
\hline & & $\rho_{\mathrm{s}}=4000 \mathrm{~kg} / \mathrm{m}^{3}$ & $\alpha_{3}=3$ \\
$\rho=1058 \mathrm{~kg} / \mathrm{m}^{3}$ & $\rho_{\mathrm{s}}=7800 \mathrm{~kg} / \mathrm{m}^{3}$ & $\kappa=5.31 \times 10^{8} \mathrm{~Pa}$ & $\mu_{1}=1.1 \times 10^{7}$ \\
$\mu=0.058 \mathrm{~kg} / \mathrm{m} \mathrm{s}$ & $E=2.07 \times 10^{11} \mathrm{~Pa}$ & $\alpha_{1}=1$ & $\mu_{2}=-6.5 \times 10^{6}$ \\
$\kappa=1.96 \times 10^{9} \mathrm{~Pa}$ & $v=0.3$ & $\alpha_{2}=2$ & $\mu_{3}=1.4 \times 10^{6}$ \\
\hline
\end{tabular}

\subsection{Axisymmetric hydraulic engine mount problem}

We consider an hydraulic engine mount subjected to excitation. Such mounts are used in the automotive industry to reduce engine vibration and noise.

Figure 4(a) shows the axisymmetric mount considered. The mount is subjected to a sinosoidal prescribed displacement at the top. The solid part consists of rubber (light gray part) and of steel at the centre (dark gray part). The fluid is oil modelled as a slightly compressible NavierStokes fluid in laminar flow conditions. Table I gives the material data used. Figure 4(b) shows the fluid mesh and the solid mesh used. The specific objective of the analysis is to predict the hysteresis behaviour of the mount for the given forced excitation. The calculated response is given in Figure 4(c) which shows that the energy absorption of the mount is significant.

\subsection{Axisymmetric high-speed flow with shock problem}

In high-speed compressible flow, the possibility and location and strength of a shock is of major concern. In internal flows, the shape of the channel plays a significant role in determining the flow field.

Figure 5(a) shows the problem considered. The shell in the channel provides for a convergingdiverging flow and hence for the possibility of a shock. In the first solution we assume that the shell is rigid. In this case the Mach contours are as given in Figure 5(b). Next, we assume that the shell is flexible. In this case the solution shown in Figure 5(c) is obtained. It is clear that the flexibility of the shell significantly affects the flow field and location of the shock.

\subsection{Collapsible channel flow problem}

This problem is of much interest in bio-mechanical studies. Figure 6(a) shows the problem considered. The decrease in the pressure at the right-hand end of the channel causes the collapsible segment of the channel wall to vibrate, and in fact hit the vertical stop (modelled as a spring). Under certain conditions, a chaotic response is observed [25].

Figure 6(b) shows the calculated mid-point displacement of the collapsible segment and Figure 6(c) gives the calculated approximation for the Lyapunov characteristic exponent. This exponent is clearly positive indicating that the response is chaotic. More details on the computations performed are given in Reference [25].

\subsection{Artificial lung problem}

The development of artificial lungs is of much interest in the field of biomedical engineering. We present here some ingredients - but no details — of the analysis of a typical artificial lung 
(a)

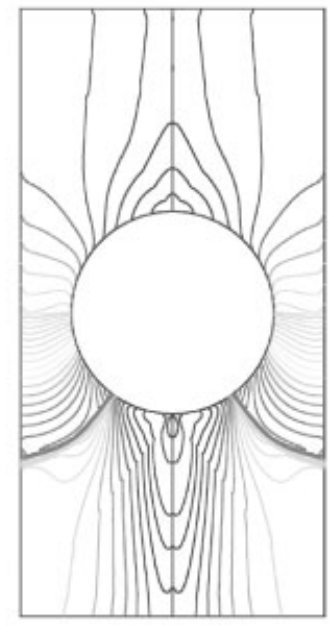

(b)

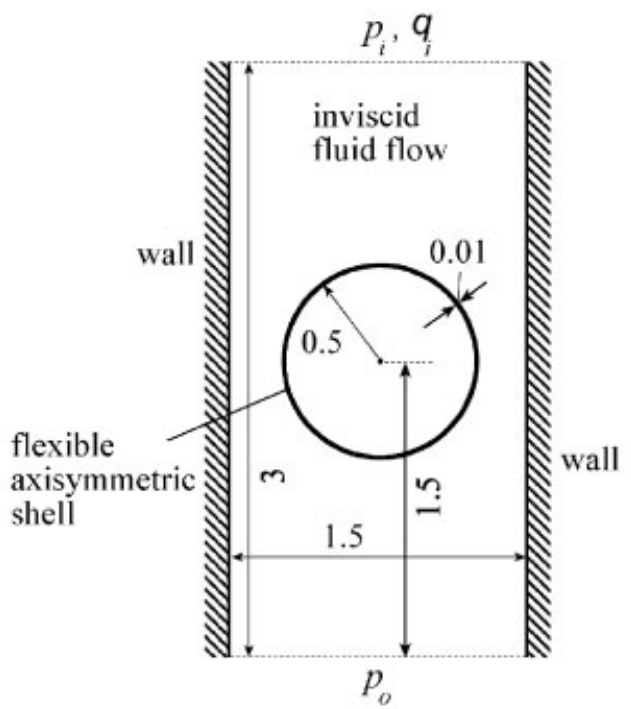

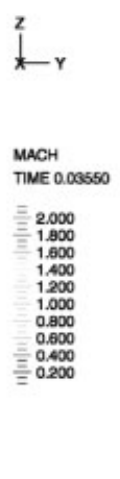

(c)

Figure 5. (a) Problem of high-speed compressible Euler fluid flow coupled with a shell. The shell properties are: $E=2.07 \times 10^{11} \mathrm{~Pa}$ and $v=0.3$. The fluid conditions and properties are: $p_{\mathrm{i}}=86100 \mathrm{~Pa} ; \theta_{\mathrm{i}}=300 \mathrm{~K} ; p_{o}=50000 \mathrm{~Pa} ; c_{p}=1004.5 \mathrm{~m}^{2} / \mathrm{s}^{2} \mathrm{~K} ;$ and $c_{v}=717.5 \mathrm{~m}^{2} / \mathrm{s}^{2} \mathrm{~K} ;(\mathrm{b})$ Mach contours from the fluid solution assuming a stiff shell; and (c) Mach contours from the fluid solution assuming a flexible shell.

problem. Figure 7(a) shows a finite element model of an artificial lung. The fluid, blood, is passed into a reservoir that is bounded by a flexible shell, modelled by four-node shell elements. The compliant shell expands to accept the fluid and then passes it by a pumping action into the core of the lung. This part of the lung is a fibre bundle which is modelled as a porous $3 \mathrm{D}$ medium. 

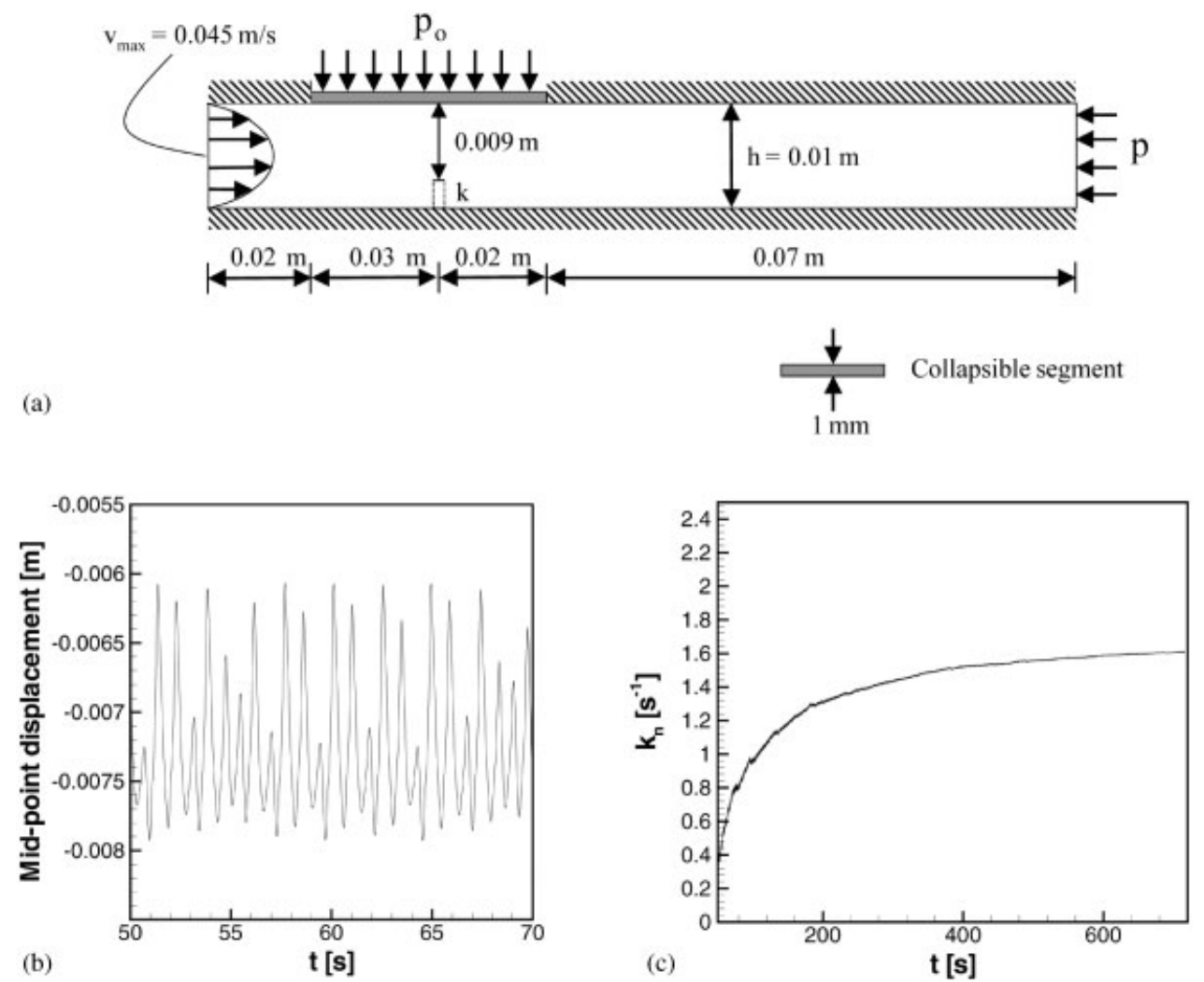

Figure 6. (a) Collapsible channel flow problem considered; (b) calculated mid-point membrane displacement; and (c) calculated Lyapunov characteristic exponent.

The interest in the numerical simulation lies in predicting the deformations of the thin shell that determine the amount of blood and oxygen that can be passed. The shell deformations are clearly dependent on the fluid pressure and, in turn, the fluid flow is influenced by the deformations of the structure. Thus the coupled solution of the fluid flow and shell becomes a key factor in analysing an artificial lung.

Typical fluid pressure and velocity results, and shell deformations drawn to scale are shown in Figure $7(b)$.

\section{CONCLUDING REMARKS}

The objective of this paper is to present some solution capabilities for fluid flow structural interaction analyses. We considered quite general flow fields and very general structural conditions, as analyzed with ADINA.

The analysis of fluid flow structural interactions in engineering practice requires a somewhat different approach than can be followed in a pure research environment. In engineering practice, 
(a)
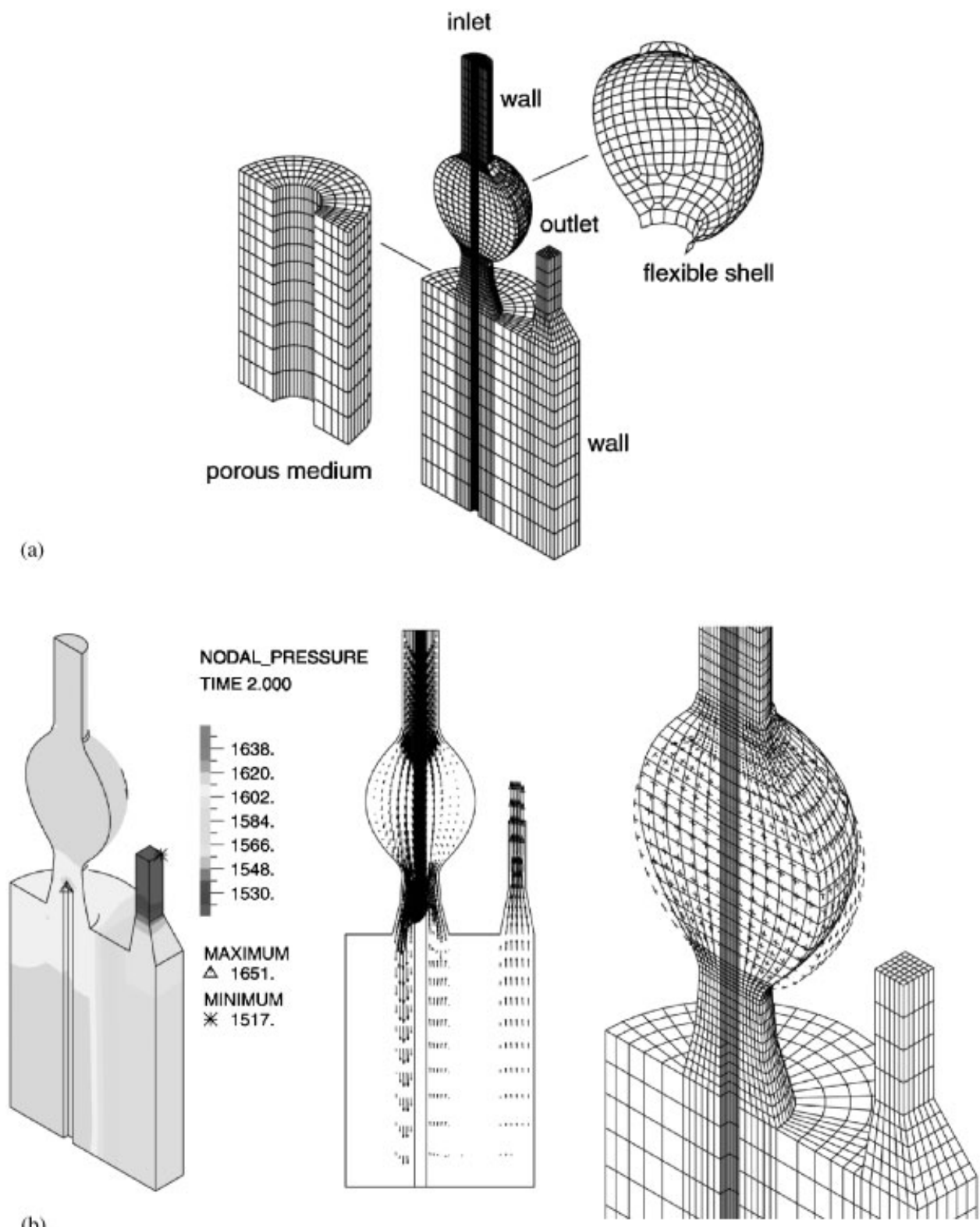

(b)

Figure 7. (a) Illustration of the artificial lung and the meshes used; and (b) typical fluid pressure (left) and velocity (centre) distributions, and the deformation of the shell drawn to scale (right, the dashed line shows the original mesh).

the analyst frequently does not know what Reynolds number flow is to be simulated. Hence, it is natural to first consider laminar flow conditions and obtain a response. Then, based on the available laminar flow solution, the analyst can choose the appropriate turbulence models to be 
used. In the paper, we thus refer to an 'ideal' solution scheme for the laminar Navier-Stokes equations. This scheme would be quite robust, reliable and accurate, not require the setting of any solution parameters, and provide good convergence in the iterative solution of the nonlinear finite element equations. Such a scheme does not exist yet, but we are developing the FCBI approach to obtain a method closer to the ideal scheme. The current FCBI procedure can already be used for complex fluid flow problems with structural interactions, and we see considerable potential to further increase its effectiveness [32], in particular when used with goal-oriented error estimations [33, 34].

There are many applications of fluid flow interaction problems and it is apparent that further significant advances in modelling and solution capabilities are in much demand. The great challenge, however, lies in the development of capabilities that are general and reliable for accurate solutions, and can be used by other persons than the code developers for the simulation of complex problems [35].

\section{REFERENCES}

1. Bathe KJ (ed.), Computational fluid and solid mechanics. Proceedings of the First M.I.T. Conference on Computational Fluid and Solid Mechanics. Elsevier Science: Amsterdam, 2001.

2. Olson LG, Bathe KJ. Analysis of fluid-structure interactions. A direct symmetric coupled formulation based on a fluid velocity potential. Computers and Structures 1985; 21:21-32.

3. Wang X, Bathe KJ. Displacement/pressure based mixed finite element formulations for acoustic fluid-structure interaction problems. International Journal for Numerical Methods in Engineering 1997; 40:2001-2017.

4. Morand HJP, Ohayon R. Fluid Structure Interaction: Applied Numerical Methods. Wiley: New York, 1995.

5. Nitikitpaiboon C, Bathe KJ. An arbitrary Lagrangian-Eulerian velocity potential formulation for fluid-structure interaction. Computers and Structures 1993; 47:871-891.

6. Sussman T, Sundqvist J. Fluid-structure interaction analysis with a subsonic potential-based fluid formulation. Computers and Structures 2003; 81:949-962.

7. Donea J, Fasoli-Stella P, Giuliani S. Lagrangian and Eulerian finite element techniques for transient fluidstructure interaction problems. Paper B1/2, Transactions of the Fourth SMIRT Conference, San Francisco, August 1977.

8. Chapelle D, Bathe KJ. Fundamental considerations for the finite element analysis of shell structures. Computers and Structures 1998; 66:19-36.

9. Bathe KJ. Finite Element Procedures. Prentice-Hall: Englewood Cliffs, NJ, 1996.

10. Hendriana D, Bathe KJ. On upwind methods for parabolic finite elements in incompressible flows. International Journal for Numerical Methods in Engineering 2000; 47:317-340.

11. Bathe KJ, Pontaza J. A flow-condition-based interpolation mixed finite element procedure for higher Reynolds number fluid flows. Mathematical Models and Methods in Applied Science 2002; 12(4):1-15.

12. Bathe KJ, Zhang H. A flow-condition-based interpolation finite element procedure for incompressible fluid flows. Computers and Structures 2002; 80:1267-1277.

13. Bathe KJ, Zhang H, Wang MH. Finite element analysis of incompressible and compressible fluid flows with free surfaces and structural interactions. Computers and Structures 1995; 56:193-213.

14. Bathe KJ, Zhang H, Zhang X. Some advances in the analysis of fluid flows. Computers and Structures 1997; 64:909-930.

15. Bathe KJ, Zhang H, Ji S. Finite element analysis of fluid flows fully coupled with structural interactions. Computers and Structures 1999; 72:1-16.

16. Rugonyi S, Bathe KJ. On finite element analysis of fluid flows fully coupled with structural interactions. Computational Modeling in Engineering Science 2001; 2:195-212.

17. El-Abbasi N, Bathe KJ. Stability and patch test performance of contact discretizations and a new solution algorithm. Computers and Structures 2001; 79:1473-1486.

18. Bathe KJ, Iosilevich A, Chapelle D. An evaluation of the MITC shell elements. Computers and Structures 2000; 75:1-30. 
19. Bathe KJ, Hiller JF, Zhang H. On the finite element analysis of shells and their full interaction with Navier-Stokes fluid flows. In Computational Structures Technology, Saxe-Coburg Publications: 2002 (Also published as a special lecture at the Croatian Academy of Arts and Sciences, Zagreb, June 2002).

20. Doering CR, Gibbon JD. Applied Analysis of the Navier-Stokes Equations. Cambridge University Press: Cambridge, 1995.

21. Foias C, Manley O, Rosa R, Temam R. Navier-Stokes Equations and Turbulence. Cambridge University Press: Cambridge, 2001.

22. Bathe KJ. The inf-sup condition and its evaluation for mixed finite element methods. Computers and Structures 2001; 79:243-252,971.

23. Bao W, Wang X, Bathe KJ. On the inf-sup condition of mixed finite element formulations for acoustic fluids. Mathematical Models and Methods in Applied Sciences 2001; 11(5):883-901.

24. Chapelle D, Bathe KJ. The Finite Element Analysis of Shells-Fundamentals. Springer: Berlin, 2003.

25. Rugonyi S, Bathe KJ. An evaluation of the Lyapunov characteristic exponent of chaotic continuous systems. International Journal for Numerical Methods in Engineering 2003; 56:145-163.

26. Rugonyi S, Bathe KJ. Lyapunov characteristic exponent calculation for finite element discretized models. Proceedings First M.I.T. Conference on Computational Fluid and Solid Mechanics. Elsevier Science: Amsterdam, 2001; 1440-1443.

27. Felippa CA, Park KC, Farhat C. Partitioned analysis of coupled systems. Computational Mechanics. In Oñate E, Idelsohn S (eds), Proceedings of the WCCM IV Conference, CIMNE, Barcelona, 1998.

28. Zhang H, Bathe KJ. Direct and iterative computing of fluid flows fully coupled with structures. Proceedings First M.I.T. Conference on Computational Fluid and Solid Mechanics. Elsevier Science: Amsterdam, 2001; 1440-1443.

29. Fernández MA, Le Tallec P. From ALE to transpiration. Proceedings First M.I.T. Conference on Computational Fluid and Solid Mechanics. Elsevier Science: Amsterdam, 2001; 1640-1643.

30. McQueen DM, Peskin CS, Zhu L. The immersed boundary method for incompressible fluid-structure interaction. Proceedings First M.I.T. Conference on Computational Fluid and Solid Mechanics. Elsevier Science, 2001; 26-30.

31. Kasagi N, Kawara S, Matsunaga A. Turbulence measurement in a separated and reattaching flow over a backward-facing step with the aid of three-dimensional particle tracking velocimetry. Proceedings of the Ninth Japan Fluid Measurement/Sixth Japan Fluid Control Joint Conference, 1991; 77-82.

32. Kohno H, Bathe KJ. Advances in the flow-condition-based interpolation procedure for advection-diffusion problems. in preparation.

33. Grätsch T, Bathe KJ. The state of a posteriori error estimation techniques for use in practical finite element analysis. Computers and Structures, in press.

34. Grätsch T, Bathe KJ. Goal-oriented error estimation in the analysis of fluid flows with structural interactions, in preparation.

35. Bathe KJ. Preface. Computational fluid and solid mechanics. Proceedings of the Second M.I.T. Conference on Computational Fluid and Solid Mechanics. Elsevier Science: Amsterdam 2003. 